\title{
Deciphering the structure, function, expression and regulation of aquaporin-5 in cancer evolution (Review)
}

\author{
LIPING WANG $^{1}$, DA HUO ${ }^{1}$, HAIYAN ZHU ${ }^{1}$, QIAN XU $^{1}$, \\ CHENGPENG GAO $^{2}$, WENFENG $\mathrm{CHEN}^{3}$ and YIXIANG ZHANG ${ }^{2}$ \\ Departments of ${ }^{1}$ Oncology, ${ }^{2}$ Respiratory, and ${ }^{3}$ Science and Education, \\ Weifang People's Hospital, Weifang, Shandong 261000, P.R. China
}

Received November 5, 2020; Accepted February 8, 2021

DOI: $10.3892 / 01.2021 .12571$

\begin{abstract}
In recent years, the morbidity rate resulting from numerous types of malignant tumor has increased annually, and the treatment of tumors has been attracting an increasing amount of attention. A number of recent studies have revealed that the water channel protein aquaporin-5 (AQP5) has become a major player in multiple types of cancer. AQP5 is abnormally expressed in a variety of tumor tissues or cells and has multiple effects on certain biological functions of tumors, such as regulating the proliferation, apoptosis and migration of tumor cells. It has been suggested that AQP5 may play an important role in the process of tumor development, opening up a new field of tumor research. The present review highlighted the structure of AQP5 and its role in tumor progression. Furthermore, the expression of AQP5 in different malignant neoplasms was summarized. In addition, the influence of not only drugs, but also different compounds on AQP5 were summarized. In conclusion, according to the findings in the present review, AQP5 has potential as a novel therapeutic target in human cancer, and other AQPs should be similarly investigated.
\end{abstract}

Correspondence to: Dr Yixiang Zhang, Department of Respiratory, Weifang People's Hospital, 151 Guangwen Road, Kuiwen, Weifang, Shandong 261000, P.R. China

E-mail: zyx654zyx654@163.com

Abbreviations: 5-FU, 5-fluorouracil; AQP5, aquaporin-5; AQPs, aquaporins; CE, Cairicoside E; CML, chronic myelogenous leukemia; CREB,cAMP-response elementbinding protein;DDP, cisplatin;EGFR, epidermal growth factor receptor; EMT, epithelial-to-mesenchymal transition; ERK, extracellular-signal-regulated kinase; MAPK, mitogen-activated protein kinase; NF- $\kappa \mathrm{B}$, nuclear factor- $\kappa \mathrm{B}$; NPA, aspartyl-proline-alanine; P-gp, P-glycoprotein 1; PDTC, pyrrolidine dithiocarbamate; Ras, reticular activating system; siRNA, small interfering RNA; TCM, Traditional Chinese Medicine. TGF- $\beta 1$, transforming growth factor $\beta 1$; TOPO II, DNA topoisomerase II

Key words: aquaporin-5, cancer, cancer therapy, drug target, drugs

\section{Contents}

1. Introduction

2. Structure of AQP5

3. Effect of AQP5 on cancer

4. Expression of AQP5 in cancer

5. Influence of drugs and compounds on AQP5

6. Conclusions and perspectives

\section{Introduction}

Aquaporins (AQPs), a family of small (30 kDa/monomer) water channel proteins that are integral membrane proteins and expressed in all living organisms, serve a vital role in water homeostasis by regulating cellular water transport. To date, a total of 13 human isoforms (AQP0-AQP12) have been found to be expressed in a cell- and tissue-dependent manner $(1,2)$. In addition to water and glycerol, several other chemical substances, such as nitrate (3), arsenite/antimony (4,5), ammonia (6), nitric oxide (7), carbon dioxide (8), hydrogen peroxide (9), urea (10), silicon (11) and anions (12), also enter and exit cells through AQPs. In recent years, AQP5 has been observed in the epithelium of various types of tissue, including that of the lacrimal (13), salivary (14) and airway submucosal glands (15), as well as type I lung cells (16) and pancreatic cells (17). As AQP5 has been observed in various pathological conditions, there has been an increasing amount of interest in its implication in carcinogenesis. An increasing number of studies $(18,19)$ have shown that AQP5 is abundantly expressed in different types of tumors, such as respiratory system tumors, digestive system tumors, reproductive system tumors, and may serve as a prognostic biomarker to assess how aggressive a cancer may be. Therefore, as AQP5 may play an important role in tumor development, it has become a novel target for antitumor therapy.

\section{Structure of AQP5}

Raina et al (20) were the first to clone AQP5 cDNA from rat submandibular gland cells and confirmed that the gene was located at the distal end of chromosome 15 in 1995. Thereafter, an increasing number of studies on AQP5 have emerged. 
AQP5 is composed of 265 amino acids and has a molecular weight of $28 \mathrm{kDa}$. However, the AQP5 protein $(27 \mathrm{kDa})$ can also be found in mouse lung tissues, and the AQP5 protein $(29 \mathrm{kDa})$ in mouse submandibular glands (21). This may be due to different tissue-specific modifications. Similar to other AQPs, AQP5 exists as a tetramer in the cell membrane, and each monomer can be used as an independent water channel. A sequence analysis revealed that the AQP5 monomer is composed of a peptide chain, which has an N-terminus and $\mathrm{C}$-terminus located on the inner side of the cell membrane (21). The peptide chain contains six $\alpha$-helix-rich tandem hydrophobic transmembrane regions and is connected by five loops (A-E loops) (21). The AQP5 is embedded in the lipid bilayer. As shown in Fig. 1, which is adapted from Raine et al (20) and Woo et al (22) as appropriate, the A, C and E rings are located outside the cell, and the $\mathrm{B}$ and $\mathrm{D}$ rings are located inside the cell. The B and E loops contain highly conserved amino acid sequences and aspartyl-proline-alanine (NPA) motifs $(20,22)$. The NPA motif is a common feature of AQP molecules and is known to be located at positions $69,70,71,185,186$ and $187(20,22)$. The B and E rings are embedded into the cell membrane at the NPA to fold into a single-pore channel with the narrowest diameter being $0.3 \mathrm{~nm}$, which the same size as a single water molecule $(20,22)$. The entire AQP5 protein is embedded into the cell membrane in an hourglass-like structure $(20,22)$. Due to the particular structure of AQP5, it serves an important role in the transmembrane transport of water.

\section{Effect of AQP5 on cancer}

The metabolic mechanisms of various tumor types are closely associated with the flow of water molecules across biofilms, and AQP-mediated fluid transport is the main way in which water enters and exits cells (1). Therefore, AQPs may serve an important role in tumor growth, invasion and metastasis (1). Furthermore, AQP5 is a vital member of the AQP family, and appears to be a potential target for oncotherapy $(18,19)$.

\section{Cellular level}

Cancer cell proliferation. A number of studies have shown that AQP5 can participate in tumorigenesis and tumor development by affecting tumor proliferation and apoptosis (23-28). AQP5 knockdown in human breast cancer cells (MCF7 cells) was associated with decreased cell proliferation (23). Zhang et al (24) reported enhanced proliferation potential in lung cancer cells with high AQP5 expression, and decreased proliferation potential in lung cancer cells with low AQP5 expression. In the aforementioned study, in mice that were injected with AQP5 cells within 2 weeks, tumor growth was found to increase significantly compared with the control group. It was also found that upregulated AQP5 was closely associated with the proliferation of digestive tumor cells, such as esophageal (25), gastric (26) and colon cancer cells (27). In a study on human chronic myelogenous leukemia (CML), it was first reported that AQP5 small interfering RNA (siRNA)-treated LAMA-84 CML cells exhibited a marked decrease in cell proliferation when compared with control siRNA-treated cells (28). Furthermore, control siRNA-treated cells exhibited a higher number of viable cells than AQP5 siRNA-treated cells (28). Therefore, these results confirmed that AQP5 can not only promote cell proliferation, but also inhibit cell apoptosis.

Cancer cell metastasis. Tumor cells can reach distant sites via the blood or lymphatic vessels, which is a phenomenon known as tumor metastasis (29). In order to determine whether AQP was associated with cell migration and its exact underlying mechanism, Chen et al (30) used short hairpin RNA to inhibit the expression of the AQP5 gene, and the results revealed that the migration potential of lung adenocarcinoma SPC-A1 cells were significantly weakened. As aforementioned, AQP5 was not only closely associated with the proliferation of breast cancer cells, but also with their migration (23). A semi-quantitative assessment demonstrated that the AQP5 labeling intensity was significantly higher in invasive carcinoma with lymph node metastasis, compared with that without lymph node metastasis (23). Furthermore, a high AQP5 expression level was associated with aggressive lymph node status and the presence of distant metastasis in esophageal (31), gastric (26), colon (32) and prostate cancer (33). In ovarian cancer, the expression of AQP5 was positively correlated with lymph node metastasis, but not associated with International Federation of Gynecology and Obstetrics stage, grade and histological type (34). A previous study demonstrated that the overexpression of AQP5 and Ki-67 was significantly associated with lymph node involvement in cervical cancer, and a positive correlation was identified between AQP5 and Ki-67 expression levels (35). Furthermore, the data also revealed that the overexpression of AQP5 and $\mathrm{Ki}-67$ was associated with a less favorable prognosis. Since it was proven by the aforementioned studies that AQP5 was associated with the ability of tumor cells to invade and metastasize, it can be concluded that AQP5 may serve as a potential novel target for to prevent tumor metastasis.

Molecular level. AQP5 and its upstream and downstream signaling pathways serve an important role in tumor proliferation, invasion and migration (19). The reticular activating system (Ras) pathway is a signaling pathway that plays an important role in the development of cancer; its basic structure is Ras/Raf/MEK/mitogen-activated protein kinase (MAPK) (36). Extracellular-signal-regulated kinase (ERK) is one of the main subfamilies of MAPK, and is associated with cell proliferation and differentiation (36). There was an experimental study which demonstrated that AQP5 was able to activate the Ras pathway and its downstream pathway via phosphorylation of the PKA consensus site in its cytoplasmic loop D, thereby directly promoting cell proliferation (37). In lung cancer cells, the proliferative ability of cells was found to be positively correlated with the expression level of AQP5 (24). At the same time, the expression levels of proliferation-associated proteins, such as proliferating cell nuclear antigen and c-Myc, were also enhanced in the cells expressing AQP5. In AQP5 cells with a high expression level, epidermal growth factor receptor (EGFR) phosphorylation was enhanced, and the ERK and MAPK signaling pathways were activated, while the activity of the EGFR/ERK/p38 MAPK pathway was decreased following the deletion of AQP5. This result was consistent with the results of the study by Yang et al (38), in which AQP5 gene silencing inhibited 


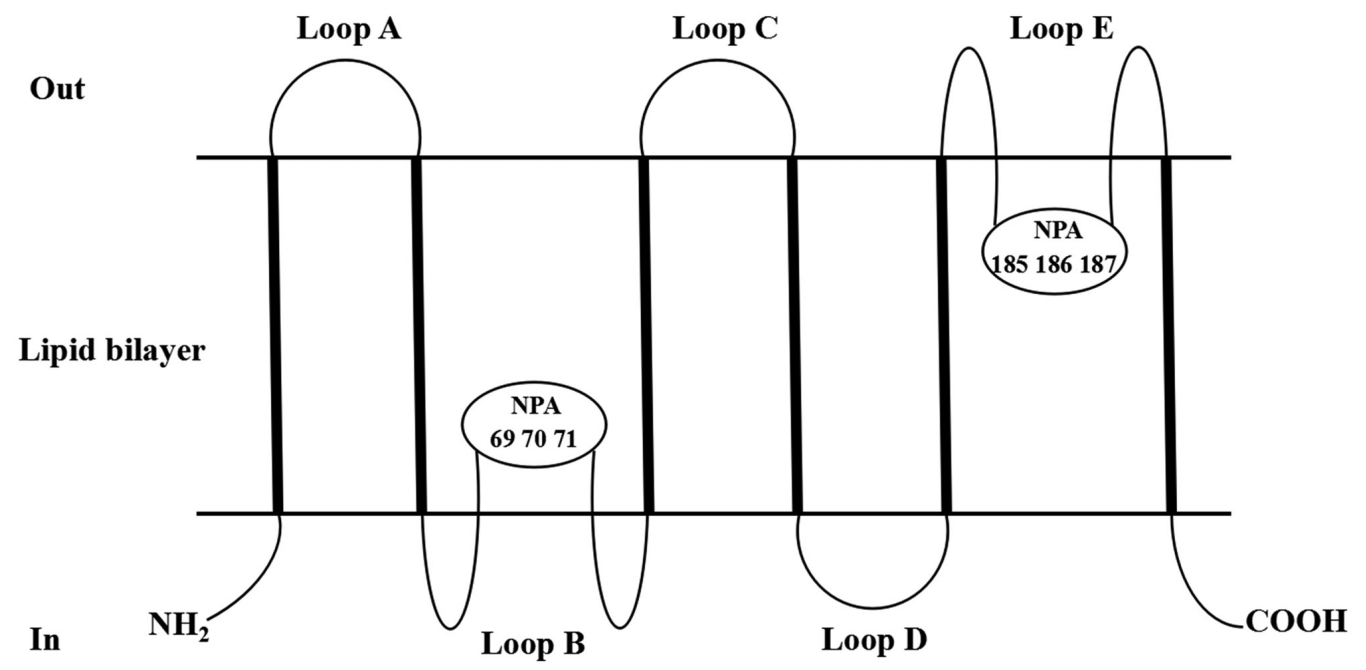

Figure 1. Structure of AQP5. This figure is adapted from Raina et al (20) and Woo et al (22) as appropriate. The AQP5 monomer is composed of a peptide chain whose N-terminal and C-terminal are located inside the cell membrane. The peptide chain is connected by five loops (A to E loop). The A, C and E rings are located outside the unit, while the B and D rings are located inside the unit. Both the B and E loops contain NPA motifs. The NPA motif is located at positions 69,70 and 71 of the B loop and 185, 186 and 187 of the E loop, respectively. AQP5, aquaporin-5; N, aspartyl; P, proline; A, alanine.

the proliferation, reduced the migration and promoted the apoptosis rates of human glioma cells by suppressing the EGFR/ERK/p38 MAPK signaling pathway. In colorectal cancer, Kang et al (39) found that the overexpression of AQP5 increased the phosphorylation of retinoblastoma $(\mathrm{Rb})$ protein, which may be achieved by activating the Ras/ERK/Rb signaling pathway.

Nuclear factor $-\kappa \mathrm{B}(\mathrm{NF}-\kappa \mathrm{B})$, as the upstream regulator of AQP5, inhibits the expression of AQP5 (40). The NF- $\kappa$ B pathway has been reported to downregulate the expression of AQP5 by inhibiting cAMP-response element binding protein (CREB) phosphorylation, or by competitive binding to the CREB-binding protein (40). Therefore, the expression levels of NF- $\mathrm{NB}$ and AQP5 are expected to be negatively correlated. However, the regulatory relationship between $\mathrm{NF}-\kappa \mathrm{B}$ and AQP5 was reversed in a CAOV3 ovarian cancer cell line (41). In the aforementioned study, a positive correlation was identified between the AQP5 protein expression and $\mathrm{NF}-\kappa \mathrm{B}$, and the growth rate of CAOV3 cells was decreased by the inhibition of the AQP5 protein. In an SKOV3 ovarian cancer cell line, the positive correlation between AQP5 and NF- $\kappa$ B was verified again (42). Pyrrolidine dithiocarbamate (PDTC), a specific blocker of $\mathrm{NF}-\kappa \mathrm{B}$, downregulated the expression of $\mathrm{NF}-\kappa \mathrm{B}$ and decreased its content in the nucleus and cytoplasm, resulting in a decrease in the mRNA and protein expression levels of AQP5 (42). At the same time, this effect also markedly decreased tumor cell proliferation (42). Therefore, these results suggested that the downregulation of AQP5 may inhibit tumor growth and be associated with $\mathrm{NF}-\kappa \mathrm{B}(42)$. In conclusion, the correlation between $\mathrm{NF}-\kappa \mathrm{B}$ and AQP5, which was reversed in certain tumors, may reflect the difference between normal and tumor tissue.

Chae et al (28) were the first to report that AKT phosphorylation was enhanced in CML cell lines overexpressing AQP5, suggesting that AQP5 may regulate tumor cell proliferation and apoptosis via the AKT signaling pathway.

Distant metastasis is the leading cause of death in patients with malignant tumors (43). Epithelial-to-mesenchymal transition (EMT) is one of the key molecular steps in distant metastasis (43). During EMT, epithelial cells lose intercellular connections and normal polarity, and acquire more mobile spindle-like interstitial phenotypes, promoting the invasion and migration of cancer cells (43). In colon cancer cells, Cairicoside E (CE), a resin glycoside isolated from Ipomoea cairica (Convolvulaceae), inhibited the migration of colon cancer cells by inhibiting EMT (44). Notably, CE had no significant effect on EMT when AQP5 was silenced (44). These results indicated that the inhibitory effect of CE on EMT depends on the downregulation of AQP5. In addition, it was found that transforming growth factor $\beta 1$ (TGF- $\beta 1$ ) promoted AQP5 expression, while AQP5-overexpression upregulated p-Smad2/3, thereby activating EMT (44). Another study demonstrated that AQP5-silencing inhibited the migration and invasion of colorectal cancer cells, which was accompanied by altered expression of EMT-associated molecules and weakened Wnt/ $\beta$-catenin signaling transduction (45). AQP5-silencing-induced changes to the cell phenotype were partly restored following the reactivation of the $\mathrm{Wnt} / \beta$-catenin pathway (45).

In this section, the regulatory effects of different AQP5-related signaling pathways on different tumors were summarized. As shown in Fig. 2, AQP5 can affect tumor cell proliferation, apoptosis and migration via multiple signaling pathways. However, whether these pathways interact with each other to participate in the regulation of tumor cell pathophysiology by AQP5 requires further investigation. Therefore, the development of therapeutic strategies using AQP5 as a drug target, although promising, requires an increased understanding of the molecular mechanisms involved in tumor biology.

\section{Expression of AQP5 in cancer}

Due to the distinct structural characteristics and abnormal expression of AQP5 in different tumor types, an increasing interest in its involvement in cancer has emerged. In tumors of 


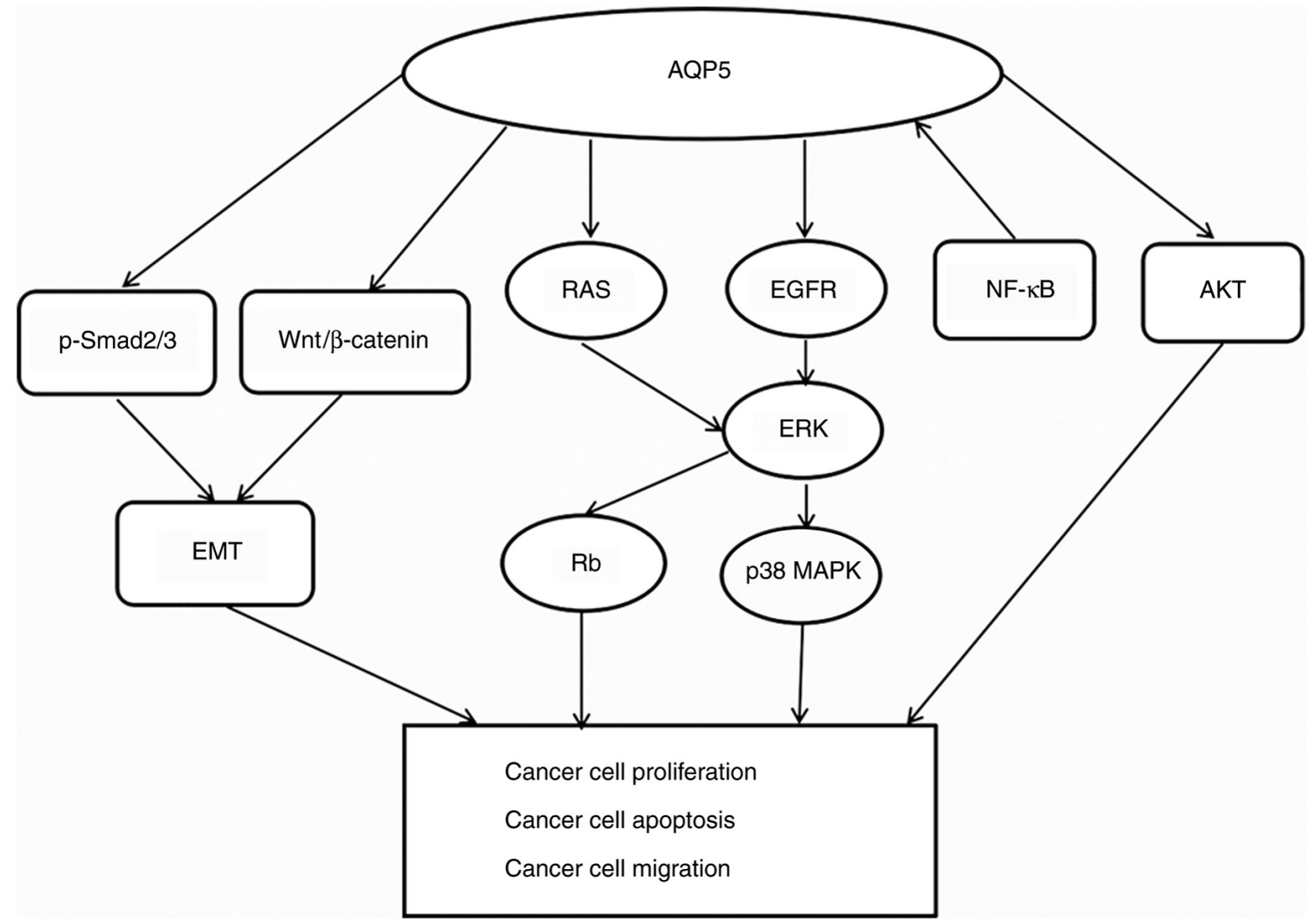

Figure 2. Signaling pathways associated with AQP5 in cancer. AQP5 is able to activate the EGFR/ERK/p38 MAPK pathway and also increase the phosphorylation of $\mathrm{Rb}$ protein by activating the Ras/ERK/Rb signaling pathway. In ovarian cancer, it was found that there was a positive correlation between AQP5 protein expression and NF- $\mathrm{KB}$. However, this is not the case in all cancer cells, as detailed in the manuscript. AQP5 can also enhance the phosphorylation of AKT to promote tumor cell proliferation. EMT is one of the key molecular steps in cancer distant metastasis. Overexpression of AQP5 can upregulate p-Smad2/3 and Wnt/ $\beta$-catenin, thereby activating EMT. In summary, AQP5 participates in tumor proliferation and migration through a variety of complex signaling pathways. AQP5, aquaporin-5; EGFR, epidermal growth factor receptor; ERK, extracellular-signal-regulated kinase; MAPK, mitogen-activated protein kinase; NF- $\mathrm{KB}$, nuclear factor- $\mathrm{\kappa B}$; Rb, retinoblastoma protein; Ras, reticular activating system; EMT, epithelial-mesenchymal transition; TGF- $\beta$, tumor growth factor- $\beta ;$ p phosphorylated.

the digestive system, it was found that AQP5 was upregulated in tongue (46), esophageal (25), gastric $(26,47)$, liver (48), bile duct (49) and colon cancer types $(26,37,39)$, but was downregulated in salivary gland adenoid cystic carcinoma (46). In ovarian tumors, Yang et al (34) reported that the expression of AQP5 was associated with the pathological characteristics of the tumor. It was found that the expression level of AQP5 in malignant and borderline ovarian tumors was significantly higher than that in benign tumors and normal tissues. Secondly, the subcellular localization of AQP5 was also different between benign and malignant tumors. AQP5 was primarily located in the basal membrane of benign tumors, as well as the parietal membrane and basal membrane of borderline tumors, and was scattered at the plasma membrane of malignant tumor cells. In addition, AQP5 expression was found to be increased in cervical (35) and endometrial cancer (50). In lung cancer (51), patients with a positive AQP5 expression had a higher tumor recurrence rate and shorter tumor-free survival time than those with a negative AQP5 expression. However, Machida et al (52) reported that the expression level of AQP5 was positively correlated with the degree of cell differentiation, but not prognosis. Therefore, the effect of AQP5 on the prognosis of lung cancer needs to be further studied. AQP5 overexpression was found to be associated with metastasis, poor prognosis, higher tumor grade and tumor recurrence in breast cancer $(23,53)$. In addition to the aforementioned studies, there are some other studies on the expression of AQP5 in malignant tumor tissues. AQP5 was found to be upregulated in primary glioblastomas and astrocytomas (54). However, due to the low number of samples tested, further research is required to confirm these preliminary findings. In prostate cancer, AQP5-overexpression was associated with Tumor-Node-Metastasis stage $(33,55)$, lymph node metastasis $(33)$ and the cumulative survival rate $(33,56)$. It can therefore be concluded that AQP5 is an independent prognostic indicator in prostate cancer.

Unlike the aforementioned studies that focused on solid tumors, to the best of our knowledge, there is currently a limited number of studies that focus on AQP5 in hematological tumors. Chae et al (28) reported that the AQP5 expression level was relatively higher in patients with $\mathrm{CML}$ diagnosed at the advanced or blast phases when compared with those diagnosed at the chronic phase, and those with a high AQP5 expression level were more likely to develop drug resistance. 


\section{Influence of drugs and compounds on AQP5}

Over the past few decades, significant progress has been made in understanding the fundamental role of AQP5 in various types of cancer. Further research on AQP5 revealed several drugs or compounds that can regulate the expression of AQP5. Therefore, AQP5 has potential as a powerful target for the development of new anticancer drugs.

Direct AQP5 inhibitors. Radiotherapy serves a vital role in the treatment of head and neck tumors, particularly nasopharyngeal carcinoma. However, even after low-dose radiotherapy, most patients still develop sinusitis, mucositis, bronchitis, pneumonia, fibrosis or soft tissue necrosis. AQP5 is the main AQP in the airway mucosa, and the rate-limiting barrier in mucin production $(57,58)$. The expression of AQP5 is closely associated with cilia function (59) and bronchoconstriction $(60,61)$. To the best of our knowledge, Liu et al (62) were the first to report that ovatodiolide can enhance AQP5 membrane transport and reverse the inhibitory effect of histamine on CREB phosphorylation, thereby reducing the side effects of radiotherapy on nasopharyngeal carcinoma. Therefore, they inferred that treatment with ovatodiolide is expected to restore normal nasopharyngeal physiology in patients with nasopharyngeal carcinoma following radiotherapy. Acetazolamide is an AQP inhibitor that is able to decrease AQP expression and AQP-related functions $(63,64)$. Huang et al $(26)$ reported that the expression of AQP5 was significantly increased in gastric cancer tissues, and the inhibition of AQP5 expression by acetazolamide decreased the proliferative and invasive abilities of gastric cancer cells. In addition, AQP5 was involved in the differentiation of gastric cancer cells. The expression of AQP5 was significantly upregulated in intestinal gastric cancer cells, while that of AQP5 was not identified in intestinal metaplasia and the diffuse type of the gastric cancer tissues. Treatment of MKN45 gastric cancer cells that stably expressed AQP5 with $\mathrm{HgCl}$, an inhibitor of AQPs, significantly decreased the proportion of differentiated cells and the activity of alkaline phosphatase (65). Heavy metal compounds are certainly effective AQP inhibitors, but their toxicity and lack of specificity substantially limit their therapeutic potential.

Direct modulation of AQP5, even by therapeutically unpalatable heavy metal ions, are the best demonstration that AQP5 inhibiting drugs can have a therapeutic effect in specific tumor types. By studying these direct regulators of AQP5, it may be possible to provide new development directions for tumor targeted therapy.

Genetic (siRNA) silencing of AQP5 expression. Chemotherapy is one of the main treatments for advanced cancer; however, its effectiveness is limited (66). One of the main reasons for that is the drug resistance of tumor cells. Multidrug resistance can be divided into two categories (66). The first is the classic drug resistance mechanism, involving energy-dependent drug pumps, which is mediated by drug-resistant membrane glycoproteins, including P-glycoprotein 1 (P-gp), multidrug resistance-associated protein 2 and resistance-related protein (66). The other is a resistance mechanism involving resistance enzymes, such as DNA topoisomerase II (TOPO II), thymidylate synthase (TS) and protein kinase C (66). AQP5 expression was found to be increased in human colon cancer tissues and was positively correlated with P-gp, glutathione S-transferase Pi and TOPO II (27). Following the use of siRNA-mediated genes to silence AQP5 expression in HT-29 colon cancer cell lines, the sensitivity of these cells to chemotherapy drugs cisplatin (DDP) and 5-fluorouracil (5-FU) was increased (27). In addition, resistance to imatinib mesylate, a tyrosine-kinase inhibitor used in CML treatment at the chronic phase, was also associated with higher levels of AQP5 expression (28). siRNA targeting AQP5 reduced the cell proliferation rate in CML cells.

The effectiveness of certain Traditional Chinese Medicine (TCM) agents in the treatment of diseases has been widely accepted. Youyou Tu, chief scientist, Institute of Chinese Materia Medica China Academy of Chinese Medical Sciences Beijing (Beijing, China), received the Nobel Prize in Physiology or Medicine in 2015 for her discovery of artemisinin, a drug used to treat malaria, which saved millions of lives around the world (67). In recent years, certain natural plant-derived compounds have attracted increasing attention in the field of antitumor drug development. The Realgar-Indigo naturalis formula has been proven to be effective in treating human acute promyelocytic leukemia (68). Furthermore, CE, a resin glycoside isolated from Ipomoea cairica, was found to be cytotoxic to tumor cells in a study by $\mathrm{Yu}$ et al (69). A follow-up study revealed that CE had no significant effect on EMT markers and p-Smad2/3 following AQP5 silencing, indicating that CE may also inhibit the EMT process by downregulating AQP5 in CRC cells, thereby inhibiting the metastasis of cancer cells (44). In summary, gene silencing of AQP5 by siRNA is also a reasonable method to directly inhibit AQP5. If certain drugs can suppress tumor growth by silencing the AQP5 gene, this may represent a major advancement for antitumor therapies.

Indirect effectors of AQP5 expression. Ovarian cancer appears to be associated with AQP5. Epigallocatechin gallate (a potential cancer drug), PDTC (an NF- $\kappa$ B specific inhibitor) and DDP (first-line drug in the defense against cancer), all inhibit proliferation and induce apoptosis of ovarian cancer cells by downregulating AQP5 expression $(41,42)$; These drugs can downregulate AQP5, which might all be associated with the nuclear transcription factor NF- $\mathrm{KB}$. It has been reported that hyperosmotic stress induced by sorbitol treatment reduced AQP5 expression in breast cancer MCF7 cells, which was also associated with a significant reduction in cell proliferation and migration (23).

In addition, there are certain physical environments that can exert antitumor effects by activating the expression of AQP5. A previous study has shown that the hypotonic solution at low temperature increases the initial water influx by activating AQP5, thereby enhancing the cytocidal effect on gastric cancer cells (70). Therefore, low-temperature lavage under hypotonic conditions may have the potential to become a new method of reducing the peritoneal recurrence of gastric cancer following radical surgery. As mentioned above drugs and certain physical environments can suppress tumors by regulating the expression of AQP5, which may not be a strong evidence in the development of antitumor drugs $(23,41,42,70)$, However, it also provides new evidence for AQP5 to become a target and prognostic indicator of antitumor therapy.

In conclusion, multiple substances can affect the regulatory effect of AQP5 on tumors. The substances include TCM and 
western medicine agents, as well as compounds. A previous study (71) showed that puerarin and 5-FU have a significant synergistic effect on gastric cancer cells. Puerarin can enhance the antiproliferative effect of 5-FU and decrease the dose required for 5-FU treatment without increasing toxicity. It was therefore hypothesized that combining TCM and western medicine may achieve more favorable anticancer effects than each of them alone, by synergistically inhibiting AQP5. That is to say, this combination could not only enhance the therapeutic effect, but also decrease the adverse side effects of various drugs. Of course, the exact method of effectively combining TCM and western medicine to maximize the therapeutic effect is a very complex subject that requires further study.

\section{Conclusions and perspectives}

The incidence of cancer has been increasing annually due to the pressure of fast-paced life, environmental pollution, bad living habits, such as smoking, alcoholism, etc. In order to improve cancer treatment, a large number of targeted studies are constantly being performed. After establishing the anticancer effects of AQP1, there has been considerable interest in the development of other AQPs for use in antitumor therapy (29). A number of studies have proven that AQP5 can be an effective therapeutic target against cancer. In the present study, the structural characteristics of AQP5 were first briefly described. Secondly, the effects of AQP5 and its expression in different tumor types were summarized. In addition, a comprehensive summary of drugs and compounds that can affect the antitumor effect of AQP5 was provided. AQP5 participates in all stages of malignant tumors, including tumor occurrence, development, recurrence, metastasis, drug resistance and prognosis. Although several potential AQP modulators have been identified, discovering better modulators, including those that have the ability to target specificity, is not without its challenges (72). In addition, the exact molecular mechanism underlying the role of AQP5 in tumors has not yet been fully elucidated. To the best of our knowledge, a genetic link between AQP5 and any known tumor types has not yet been identified. This may be due to the current research on AQP5 being relatively new. Therefore, more comprehensive and in-depth research is required to build on these findings. In conclusion, with the continuous increase in research on AQP5, the investigation into various treatment methods based on the expression and regulation of AQP5, with the aim of intervening in the biological behavior of tumors, will provide new ideas and methods for the clinical treatment of cancer.

\section{Acknowledgements}

Not applicable.

\section{Funding}

No funding was received.

\section{Availability of data and materials}

Not applicable.

\section{Authors' contributions}

LW collected and analysed the data, and was a major contributor in drafting the original manuscript. DH, HZ, QX, $\mathrm{CG}$ and WC collected and analysed the data. YZ reviewed and edited the manuscript. LW and YZ confirm the authenticity of all the raw data. All authors have read and approved the final manuscript.

\section{Ethics approval and consent to participate}

Not applicable.

\section{Patient consent for publication}

Not applicable.

\section{Competing interests}

The authors declare that they have no competing interests.

\section{References}

1. Takata K, Matsuzaki T and Tajika Y: Aquaporins: Water channel proteins of the cell membrane. Prog Histochem Cytochem 39: $1-83,2004$

2. Ishibashi K, Tanaka Y and Morishita Y: The role of mammalian superaquaporins inside the cell. Biochim Biophys Acta 1840: 1507-1512, 2014

3. Ikeda M, Beitz E, Kozono D, Guggino WB, Agre P and Yasui M: Characterization of aquaporin- 6 as a nitrate channel in mammalian cells. Requirement of pore-lining residue threonine 63. J Biol Chem 277: 39873-39879, 2002.

4. Sanders OI, Rensing C, Kuroda M, Mitra B and Rosen BP: Antimonite is accumulated by the glycerol facilitator GlpF in Escherichia coli. J Bacteriol 179: 3365-3367, 1997.

5. Liu Z, Shen J, Carbrey JM, Mukhopadhyay R, Agre P and Rosen BP: Arsenite transport by mammalian aquaglyceroporins AQP7 and AQP9. Proc Natl Acad Sci USA 99: 6053-6058, 2002.

6. Holm LM, Jahn TP, Møller AL, Schjoerring JK, Ferri D, Klaerke DA and Zeuthen T: $\mathrm{NH} 3$ and $\mathrm{NH}_{4}{ }^{+}$permeability in aquaporin-expressing Xenopus oocytes. Pflugers Arch 450: 415-428, 2005 .

7. Herrera M, Hong NJ and Garvin JL: Aquaporin-1 transports NO across cell membranes. Hypertension 48: 157-164, 2006.

8. Nakhoul NL, Davis BA, Romero MF and Boron WF: Effect of expressing the water channel aquaporin- 1 on the $\mathrm{CO}_{2}$ permeability of Xenopus oocytes. Am J Physiol 274: C543-C548, 1998.

9. Bienert GP, Møller AL, Kristiansen KA, Schulz A, Møller IM, Schjoerring JK and Jahn TP: Specific aquaporins facilitate the diffusion of hydrogen peroxide across membranes. J Biol Chem 282: 1183-1192, 2007.

10. Ma T, Yang B and Verkman AS: Cloning of a novel water and urea-permeable aquaporin from mouse expressed strongly in colon, placenta, liver, and heart. Biochem Biophys Res Commun 240: 324-328, 1997.

11. Ma JF, Tamai K, Yamaji N, Mitani N, Konishi S, Katsuhara M, Ishiguro M, Murata Y and Yano M: A silicon transporter in rice. Nature 440: 688-691, 2006.

12. Yasui M, Hazama A, Kwon TH, Nielsen S, Guggino WB and Agre P: Rapid gating and anion permeability of an intracellular aquaporin. Nature 402: 184-187, 1999.

13. Gresz V, Kwon TH, Gong H, Agre P, Steward MC, King LS and Nielsen S: Immunolocalization of AQP-5 in rat parotid and submandibular salivary glands after stimulation or inhibition of secretion in vivo. Am J Physiol Gastrointest Liver Physiol 287: G151-G161, 2004.

14. Krane CM, Melvin JE, Nguyen HV, Richardson L, Towne JE, Doetschman T and Menon AG: Salivary acinar cells from aquaporin 5-deficient mice have decreased membrane water permeability and altered cell volume regulation. J Biol Chem 276: 23413-23420, 2001. 
15. Kreda SM, Gynn MC, Fenstermacher DA, Boucher RC and Gabriel SE: Expression and localization of epithelial aquaporins in the adult human lung. Am J Respir Cell Mol Biol 24: 224-234, 2001.

16. Dobbs LG, Gonzalez R, Matthay MA, Carter EP, Allen L and Verkman AS: Highly water-permeable type I alveolar epithelial cells confer high water permeability between the airspace and vasculature in rat lung. Proc Natl Acad Sci USA 95: 2991-2996, 1998.

17. Burghardt B, Elkaer ML, Kwon TH, Rácz GZ, Varga G, Steward MC and Nielsen S: Distribution of aquaporin water channels AQP1 and AQP5 in the ductal system of the human pancreas. Gut 52: 1008-1016, 2003.

18. Moosavi MS and Elham Y: Aquaporins 1, 3 and 5 in Different Tumors, their Expression, Prognosis Value and Role as New Therapeutic Targets. Pathol Oncol Res 26: 615-625, 2020.

19. Direito I, Madeira A, Brito MA and Soveral G: Aquaporin-5: From structure to function and dysfunction in cancer. Cell Mol Life Sci 73: 1623-1640, 2016

20. Raina S, Preston GM, Guggino WB and Agre P: Molecular cloning and characterization of an aquaporin cDNA from salivary, lacrimal, and respiratory tissues. J Biol Chem 270: 1908-1912, 1995.

21. Horsefield R, Nordén K, Fellert M, Backmark A Törnroth-Horsefield S, Terwisscha van Scheltinga AC, Kvassman J, Kjellbom P, Johanson U and Neutze R: High-resolution X-ray structure of human aquaporin 5. Proc Natl Acad Sci USA 105: $13327-13332,2008$

22. Woo J, Lee J, Chae YK, Kim MS, Baek JH, Park JC, Park MJ, Smith IM, Trink B, Ratovitski E, et al: Overexpression of AQP5, a putative oncogene, promotes cell growth and transformation. Cancer Lett 264: 54-62, 2008

23. Jung HJ, Park JY, Jeon HS and Kwon TH: Aquaporin-5: A marker protein for proliferation and migration of human breast cancer cells. PLoS One 6: e28492, 2011.

24. Zhang Z, Chen Z, Song Y, Zhang P, Hu J and Bai C: Expression of aquaporin 5 increases proliferation and metastasis potential of lung cancer. J Pathol 221: 210-220, 2010.

25. Shimizu H, Shiozaki A, Ichikawa D, Fujiwara H, Konishi H, Ishii H, Komatsu S, Kubota T, Okamoto K, Kishimoto M, et al: The expression and role of Aquaporin 5 in esophageal squamous cell carcinoma. J Gastroenterol 49: 655-666, 2014.

26. Huang YH, Zhou XY, Wang HM, Xu H, Chen J and Lv NH: Aquaporin 5 promotes the proliferation and migration of human gastric carcinoma cells. Tumour Biol 34: 1743-1751, 2013.

27. Shi X, Wu S, Yang Y, Tang L, Wang Y, Dong J, Lü B, Jiang G and Zhao W: AQP5 silencing suppresses p38 MAPK signaling and improves drug resistance in colon cancer cells. Tumour Biol 35: 7035-7045, 2014

28. Chae YK, Kang SK, Kim MS, Woo J, Lee J, Chang S, Kim DW, Kim M, Park S, Kim I, et al: Human AQP5 plays a role in the progression of chronic myelogenous leukemia (CML). PLoS One 3: e2594, 2008

29. Wang L, Zhang Y, Wu X and Yu G: Aquaporins: New Targets for Cancer Therapy. Technol Cancer Res Treat 15: 821-828, 2016.

30. Chen Z, Zhang Z, Gu Y and Bai C: Impaired migration and cell volume regulation in aquaporin 5-deficient SPC-A1 cells. Respir Physiol Neurobiol 176: 110-117, 2011.

31. Liu S, Zhang S, Jiang H, Yang Y and Jiang Y: Co-expression of AQP3 and AQP5 in esophageal squamous cell carcinoma correlates with aggressive tumor progression and poor prognosis. Med Oncol 30: 636, 2013.

32. Kang BW, Kim JG, Lee SJ, Chae YS, Jeong JY, Yoon GS, Park SY, Kim HJ, Park JS, Choi GS and Jeong JY: Expression of aquaporin-1, aquaporin-3, and aquaporin-5 correlates with nodal metastasis in colon cancer. Oncology 88: 369-376, 2015.

33. Li J, Wang Z, Chong T, Chen H, Li H, Li G, Zhai X and Li Y: Over-expression of a poor prognostic marker in prostate cancer: AQP5 promotes cells growth and local invasion. World J Surg Oncol 12: 284, 2014

34. Yang JH, Shi YF, Cheng Q and Deng L: Expression and localization of aquaporin-5 in the epithelial ovarian tumors. Gynecol Oncol 100: 294-299, 2006.

35. Zhang T, Zhao C, Chen D and Zhou Z: Overexpression of AQP5 in cervical cancer: Correlation with clinicopathological features and prognosis. Med Oncol 29: 1998-2004, 2012

36. Santarpia L, Lippman SM and El-Naggar AK: Targeting the MAPK-RAS-RAF signaling pathway in cancer therapy. Expert Opin Ther Targets 16: 103-119, 2012.
37. Woo J, Lee J, Kim MS, Jang SJ, Sidransky D and Moon C: The effect of aquaporin 5 overexpression on the Ras signaling pathway. Biochem Biophys Res Commun 367: 291-298, 2008

38. Yang J,Zhang JN, Chen WL, Wang GS, Mao Q, Li SQ, Xiong WH, Lin YY, Ge JW, Li XX, et al: Effects of AQP5 gene silencing on proliferation, migration and apoptosis of human glioma cells through regulating EGFR/ERK/p38 MAPK signaling pathway. Oncotarget 8: 38444-38455, 2017.

39. Kang SK, Chae YK, Woo J, Kim MS, Park JC, Lee J, Soria JC, Jang SJ, Sidransky D and Moon C: Role of human aquaporin 5 in colorectal carcinogenesis. Am J Pathol 173: 518-525, 2008.

40. Wang W and Zheng M: Nuclear factor kappa B pathway down-regulates aquaporin 5 in the nasal mucosa of rats with allergic rhinitis. Eur Arch Otorhinolaryngol 268: 73-81, 2011.

41. Yang J, Yan C, Zheng W and Chen X: Proliferation inhibition of cisplatin and aquaporin 5 expression in human ovarian cancer cell CAOV3. Arch Gynecol Obstet 285: 239-245, 2012.

42. Yan C, Yang J, Shen L and Chen X: Inhibitory effect of Epigallocatechin gallate on ovarian cancer cell proliferation associated with aquaporin 5 expression. Arch Gynecol Obstet 285: 459-467, 2012.

43. Tsai JH and Yang J: Epithelial-mesenchymal plasticity in carcinoma metastasis. Genes Dev 27: 2192-2206, 2013.

44. Chen C, Ma T, Zhang C, Zhang H, Bai L, Kong L and Luo J: Down-regulation of aquaporin 5-mediated epithelial-mesenchymal transition and anti-metastatic effect by natural product Cairicoside $\mathrm{E}$ in colorectal cancer. Mol Carcinog 56: 2692-2705, 2017.

45. Wang W, Li Q, Yang T, Li D, Ding F, Sun H and Bai G: Anti-cancer effect of Aquaporin 5 silencing in colorectal cancer cells in association with inhibition of $\mathrm{Wnt} / \beta$-catenin pathway. Cytotechnology 70: 615-624, 2018

46. Ishimoto S, Wada K, Usami Y, Tanaka N, Aikawa T, Okura M, Nakajima A, Kogo M and Kamisaki Y: Differential expression of aquaporin 5 and aquaporin 3 in squamous cell carcinoma and adenoid cystic carcinoma. Int J Oncol 41: 67-75, 2012.

47. Tan SH, Swathi Y, Tan S, Goh J, Seishima R, Murakami K, Oshima M, Tsuji T, Phuah P, Tan LT, et al: AQP5 enriches for stem cells and cancer origins in the distal stomach. Nature 578: 437-443, 2020

48. Guo X, Sun T, Yang M, Li Z, Li Z and Gao Y: Prognostic value of combined aquaporin 3 and aquaporin 5 overexpression in hepatocellular carcinoma. BioMed Res Int 2013: 206525, 2013.

49. Sekine S, Shimada Y, Nagata T, Moriyama M, Omura T, Watanabe T, Hori R, Yoshioka I, Okumura T, Sawada S, et al: Prognostic significance of aquaporins in human biliary tract carcinoma. Oncol Rep 27: 1741-1747, 2012.

50. Jiang XX, Xu KH, Ma JY, Tian YH, Guo XY, Lin J and Wu RJ Reduced migration of Ishikawa cells associated with downregulation of aquaporin-5. Oncol Lett 4: 257-261, 2012.

51. Chae YK, Woo J, Kim MJ, Kang SK, Kim MS, Lee J, Lee SK, Gong G, Kim YH, Soria JC, et al: Expression of aquaporin 5 (AQP5) promotes tumor invasion in human non small cell lung cancer. PLoS One 3: e2162, 2008.

52. Machida Y, Ueda Y, Shimasaki M, Sato K, Sagawa M, Katsuda S and Sakuma T: Relationship of aquaporin 1,3, and 5 expression in lung cancer cells to cellular differentiation, invasive growth, and metastasis potential. Hum Pathol 42: 669-678, 2011.

53. Shi Z, Zhang T, Luo L, Zhao H, Cheng J, Xiang J and Zhao C: Aquaporins in human breast cancer: Identification and involvement in carcinogenesis of breast cancer. J Surg Oncol 106: 267-272, 2012.

54. McCoy E and Sontheimer H: Expression and function of water channels (aquaporins) in migrating malignant astrocytes. Glia 55: 1034-1043, 2007

55. Lee H, Lee M, Byun SS, Lee SE and Hong SK: Evaluation of Prostate Cancer Stage Groups Updated in the 8th Edition of the American Joint Committee on Cancer Tumor-Node-Metastasis Staging Manual. Clin Genitourin Cancer 17: e221-e226, 2019.

56. Pust A, Kylies D, Hube-Magg C, Kluth M, Minner S, Koop C, Grob T, Graefen M, Salomon G, Tsourlakis MC, et al: Aquaporin 5 expression is frequent in prostate cancer and shows a dichotomous correlation with tumor phenotype and PSA recurrence. Hum Pathol 48: 102-110, 2016.

57. Hansel NN, Sidhaye V, Rafaels NM, Gao L, Gao P, Williams R, Connett JE, Beaty TH, Mathias RA, Wise RA, et al: Aquaporin 5 polymorphisms and rate of lung function decline in chronic obstructive pulmonary disease. PLoS One 5: e14226, 2010.

58. Shen Y, Wang Y, Chen Z, Wang D, Wang X, Jin M and Bai C: Role of aquaporin 5 in antigen-induced airway inflammation and mucous hyperproduction in mice. J Cell Mol Med 15: 1355-1363, 2011. 
59. Shikani AH, Sidhaye VK, Basaraba RJ, Shikani HJ, Alqudah MA, Kirk N, Cope E and Leid JG: Mucosal expression of aquaporin 5 and epithelial barrier proteins in chronic rhinosinusitis with and without nasal polyps. Am J Otolaryngol 35: 377-383, 2014.

60. Krane CM, Fortner CN, Hand AR, McGraw DW, Lorenz JN Wert SE, Towne JE, Paul RJ, Whitsett JA and Menon AG: Aquaporin 5-deficient mouse lungs are hyperresponsive to cholinergic stimulation. Proc Natl Acad Sci USA 98: 14114-14119, 2001.

61. Aggarwal NR, Chau E, Garibaldi BT, Mock JR, Sussan T, Rao K, Rao K, Menon AG, D'Alessio FR, Damarla M, et al: Aquaporin 5 regulates cigarette smoke induced emphysema by modulating barrier and immune properties of the epithelium. Tissue Barriers 1: e25248, 2013.

62. Liu SC, Huang CM, Chang YL, Bamodu OA, Yeh CT, Wang HW, Lee FP and Lin CS: Ovatodiolide suppresses inflammatory response in BEAS-2B cells by regulating the CREB/AQP5 pathway, and sensitizes nasopharyngeal carcinoma cells to radiation therapy. Eur J Pharmacol 859: 172548, 2019.

63. Ameli PA, Madan M, Chigurupati S, Yu A, Chan SL and Pattisapu JV: Effect of acetazolamide on aquaporin-1 and fluid flow in cultured choroid plexus. Acta Neurochir Suppl (Wien) 113: 59-64, 2012

64. Tanimura Y, Hiroaki Y and Fujiyoshi Y: Acetazolamide reversibly inhibits water conduction by aquaporin-4. J Struct Biol 166: 16-21, 2009.
65. Watanabe T, Fujii T, Oya T, Horikawa N, Tabuchi Y, Takahashi Y, Morii M, Takeguchi N, Tsukada K and Sakai H: Involvement of aquaporin-5 in differentiation of human gastric cancer cells. J Physiol Sci 59: 113-122, 2009.

66. Beck WT: The cell biology of multiple drug resistance. Biochem Pharmacol 36: 2879-2887, 1987.

67. Mikić D: The 2015 Nobel Prize Laureates in Physiology or Medicine. Vojnosanit Preg1 72: 951-952, 2015.

68. Wang L, Zhou GB, Liu P, Song JH, Liang Y, Yan XJ, Xu F, Wang BS, Mao JH, Shen ZX, et al: Dissection of mechanisms of Chinese medicinal formula Realgar-Indigo naturalis as an effective treatment for promyelocytic leukemia. Proc Natl Acad Sci USA 105: 4826-4831, 2008

69. Yu B, Luo J, Wang J, Zhang D, Yu S and Kong L: Pentasaccharide resin glycosides from Ipomoea cairica and their cytotoxic activities. Phytochemistry 95: 421-427, 2013.

70. Shiozaki A, Yamazato Y, Kosuga T, Kudou M, Shoda K, Arita T, Konishi H, Komatsu S, Kubota T, Fujiwara H, et al: Effect of low temperature on the regulation of cell volume after hypotonic shock in gastric cancer cells. Int J Oncol 55: 905-914, 2019.

71. Guo XF, Yang ZR, Wang J, Lei XF, Lv XG and Dong WG: Synergistic antitumor effect of puerarin combined with 5-fluorouracil on gastric carcinoma. Mol Med Rep 11: 2562-2568, 2015.

72. Verkman AS, Anderson MO and Papadopoulos MC: Aquaporins: Important but elusive drug targets. Nat Rev Drug Discov 13: 259-277, 2014. 